\title{
SCALAR CURVATURE AND THE THURSTON NORM
}

\author{
P. B. Kronheimer and T. S. MrowkA
}

\section{Introduction}

Let $Y$ be a closed, oriented 3 -manifold with $b_{1} \neq 0$, and suppose that $Y$ contains no non-separating 2-spheres or tori. For such a $Y$, the dual Thurston norm can be defined on $H^{2}(Y ; \mathbb{R})$ by the formula

$$
|\alpha|=\sup _{\Sigma}\langle\alpha,[\Sigma]\rangle /(2 g(\Sigma)-2)
$$

the supremum being taken over all connected, oriented surfaces $\Sigma$ embedded in $Y$ whose genus $g$ is at least 2 [8]. If $Y$ contains spheres or non-separating tori, the definition is extended by declaring that $\alpha$ has infinite norm if it has non-zero pairing with any sphere or torus. It has been noted by various authors that there is a connection between the genus of embedded surfaces and solutions of the Seiberg-Witten monopole equations. In the present context, the result can be phrased as follows. The monopole equations depend on the choice of a metric $h$ on $Y$ and a $\operatorname{Spin}^{c}$ structure $\mathfrak{c}$, to which we can associate the class $c_{1}(\mathfrak{c})$, the first Chern class of the associated spin bundle. The result then states that, if the dual Thurston norm of $c_{1}(\mathfrak{c})$ is greater than 1 , then there exists a metric $h$ on $Y$ for which the corresponding monopole equations admit no solution.

A fact that lies rather deeper is that the connection between the Thurston norm and the monopole equations is sharp, in the following sense, at least in the case that $Y$ is irreducible (that is, every sphere bounds a ball in $Y$ ). Let us say that a cohomology class $\alpha \in H^{2}(Y ; \mathbb{R})$ is a monopole class if there is a $\operatorname{Spin}^{c}$ structure $\mathfrak{c}$, with $c_{1}(\mathfrak{c})=\alpha$ over the reals, such that the corresponding monopole equations have a solution for all Riemannian metrics $h$ on $Y$. Then we have:

Theorem 1. If $Y$ is a closed, oriented, irreducible 3-manifold, then the convex hull of the monopole classes is precisely the unit ball for the dual Thurston norm on $H^{2}(Y ; \mathbb{R})$.

(The unit ball is understood to be a convex polytope lying in the linear subspace on which the norm is finite. Note also that zero is always a monopole class, because there is always a trivial solution of the equations.)

This theorem is implicit, though not stated, in the content of [4]. On the topological side, the theorem rests on Gabai's characterization of the Thurston

Received August 20, 1997.

The first author was partially supported by NSF grant number DMS-9531964

The second author was partially supported by NSF/NYI grant number DMS-9357641 
norm in terms of taut foliations [2], together with the relationship between foliations and contact structures described in [1]. On the gauge theory side, the ingredients are drawn from Taubes' work on symplectic 4-manifolds in [6, 7], as adapted to the setting of 4-manifolds with contact boundary in [4].

One purpose of the present paper is to make explicit the proof of Theorem 1. We shall also draw the following corollary, which characterizes the dual Thurston norm in terms which make no mention of the monopole equations. For each metric $h$, one obtains a norm $\|\alpha\|_{h}$ on $H^{2}(X ; \mathbb{R})$ from the $L^{2}$ norm of the harmonic representative. Since 2 -forms scale in the same way as curvature, we can divide $\|\alpha\|_{h}$ by the $L^{2}$ norm of the scalar curvature $s_{h}$ to obtain a scale-invariant quantity:

$$
\|\alpha\|_{h} /\left\|s_{h}\right\|_{h} .
$$

Our result states that the unit ball of the dual Thurston norm consists of the classes $\alpha$ whose $L^{2}$ norm is no larger than the $L^{2}$ norm of $s_{h} / 4 \pi$ for all metrics $h$ :

Theorem 2. Let $Y$ be a closed, oriented, irreducible 3-manifold. Then the dual Thurston norm of $\alpha \in H^{2}(Y ; \mathbb{R})$ is given by

$$
|\alpha|=4 \pi \sup _{h}\|\alpha\|_{h} /\left\|s_{h}\right\|_{h}
$$

the supremum being taken over all Riemannian metrics on $Y$.

In this theorem, it is quite easy to see that the right-hand side is at least as large the left-hand side; but the authors have no proof that it is not strictly larger, other than to appeal to the previous theorem and all the machinery that it contains. The same theorem is true if one replaces the $L^{2}$ norms with $C^{0}$ norms. Other corollaries in a similar spirit are given at the end of this paper. Some of these can be proved without the Seiberg-Witten monopole equations, using minimal surfaces. It seems very likely that the hypothesis of irreducibility can be removed from both theorems.

Although no such sharp results are available in four dimensions, there is something to be gained from looking at existing four-dimensional results and rephrasing their proofs so as to isolate the role of the scalar curvature. A companion paper [3] follows this line, obtaining new bounds for the genus of embedded surfaces in 4-manifolds of the form $S^{1} \times Y$.

\section{Proofs of the two theorems}

Let $\mathfrak{c}$ be a $\operatorname{Spin}^{c}$ structure on a closed, oriented 3-manifold $Y$. Given a Riemannian metric $h$, one has the associated spin bundle $W$ or $W_{\mathfrak{c}}$, a hermitian 2-plane bundle. Differential forms act as endomorphisms of $W$ through Clifford multiplication $\rho$. The monopole equations are then the following equations 
for a section $\Phi$ of $W_{\mathfrak{c}}$ and a spin connection $A$ (a spin connection is a unitary connection in $W$ which makes $\rho$ covariant constant):

$$
\begin{aligned}
\rho\left(F_{\hat{A}}\right)-\frac{1}{2}\left\{\Phi \otimes \Phi^{*}\right\} & =0 \\
D_{A} \Phi & =0 .
\end{aligned}
$$

Here $\hat{A}$ is the induced connection in the line bundle $\operatorname{det}(W)$, and $D_{A}$ is the Dirac operator coupled to $A$. The curly brackets indicate the trace-free part of the endomorphism. Our conventions follow [4].

The following argument goes back to [10]. If $(A, \Phi)$ is solution to (1), then by applying $D_{A}^{*}$ to the second equation and using the Weitzenbock formula for the Dirac operator, one obtains

$$
\nabla_{A}^{*} \nabla_{A} \Phi+\frac{s}{4} \Phi+\frac{1}{2} \rho\left(F_{\hat{A}}\right) \Phi=0
$$

where $s=s_{h}$ is the scalar curvature. After using the first equation, pairing the equation with $\Phi$ and integrating by parts, one obtains

$$
\int\left|\nabla_{A} \Phi\right|^{2}+\int \frac{s}{4}|\Phi|^{2}+\int \frac{1}{8}|\Phi|^{4}=0
$$

and hence

$$
\int|\Phi|^{4} \leq 4 \int s^{2}
$$

by Cauchy-Schwartz if $\Phi$ is not identically zero. The first equation implies that $|\Phi|^{2}=4\left|F_{\hat{A}}\right|$, so we arrive at an inequality between $L^{2}$ norms:

$$
\left\|F_{\hat{A}}\right\| \leq\|s\| / 2
$$

The 2 -form $F_{\hat{A}}$ represents the class $(2 \pi / i) \alpha$, where $\alpha=c_{1}(\mathfrak{c})$. The harmonic representative cannot have larger norm, so recalling that $\alpha$ is said to be a monopole class if a solution exists for all metrics $h$, we deduce

Lemma 3. If $\alpha \in H^{2}(Y ; \mathbb{R})$ is a monopole class, then

$$
\|\alpha\|_{h} \leq \frac{1}{4 \pi}\|s\|_{h}
$$

for all metrics $h$.

The next lemma is elementary. It is the easy half of Theorem 2.

Lemma 4. The dual Thurston norm satisfies

$$
|\alpha| \leq \sup _{h} 4 \pi\|\alpha\|_{h} /\left\|s_{h}\right\|_{h}
$$


Proof. Let $\Sigma$ be an oriented embedded surface of genus 2 or more. Let $h_{1}$ be a Riemannian metric on $Y$ such that some neighborhood of $Y$ is isometric to a product $\Sigma \times[0,1]$, with $\Sigma$ having constant negative curvature and unit area. Let $h_{r}$ be a metric which contains a product region $\Sigma \times[0, r]$ and is isometric to $h_{1}$ outside that region. Then

$$
\left\|s_{h}\right\|_{h}=4 \pi r^{1 / 2}(2 g-2)+O(1)
$$

as $r \rightarrow \infty$, while any 2-form $\omega$ representing a class $\alpha$ must satisfy

$$
\|\omega\|_{h} \geq r^{1 / 2}\langle\alpha,[\Sigma]\rangle
$$

Thus

$$
\langle\alpha,[\Sigma]\rangle /(2 g-2) \leq \sup _{h} 4 \pi\|\alpha\|_{h} /\left\|s_{h}\right\|_{h} .
$$

This is the desired result if $\alpha$ has zero pairing with all spheres and tori in $Y$.

If $\alpha$ has non-trivial pairing with a torus (and a fortiori if $\alpha$ has a non-trivial pairing with a sphere, since one may add a handle to a sphere), a similar stretching argument shows that the supremum on the right hand side is infinite: one simply introduces a long cylinder $T^{2} \times[0, r]$ with zero scalar curvature.

The next proposition is the last leg of the argument:

Proposition 5. For a closed, oriented, irreducible 3-manifold $Y$, the unit ball of the dual Thurston norm lies inside the convex hull of the monopole classes.

Proof. Given an oriented surface $\Sigma$ embedded in $Y$, having least possible genus in its homology class, Gabai proves [2] the existence of a taut foliation $\mathcal{F}$ having $\Sigma$ as a compact leaf. (A foliation of a 3-manifold by 2-dimensional leaves is taut if every leaf is met by a closed curve transverse to the leaves.) Because $\Sigma$ is a leaf, the euler class of the tangent distribution of the foliation satisfies

$$
\langle e(\mathcal{F}),[\Sigma]\rangle=2 g-2,
$$

after adjusting the orientation. The convex hull of the euler classes of these taut foliations is the unit ball for the dual Thurston norm. If $\Sigma$ is a torus, the theorem only guarantees a $C^{0}$ foliation, but in all other cases it can be made smooth. Except in the trivial case that the tori provide a basis for the homology, we can discard the non-smooth foliations, and still have a spanning set for the unit ball. We shall show that if $\mathcal{F}$ is a smooth, taut foliation, then $e(\mathcal{F})$ is a monopole class.

When $\mathcal{F}$ is any smooth, oriented foliation of $Y$, it is shown in [1] that there are contact structures on $Y$ defined by oriented 2-plane fields $\xi_{+}$and $\xi_{-}$, compatible with the given orientation of $Y$ and the opposite orientation respectively. These 2-plane fields uniformly approximate the tangent distribution of the foliation. In the case that the foliation is taut, there is in addition a symplectic form $\omega$ on the 4-manifold $X=[0,1] \times Y$ which is compatible with these two contact 
structures on the boundary components $\{1\} \times Y$ and $\{0\} \times \bar{Y}$. The compatibility condition is that $\omega$ is positive on the oriented 2-planes of the contact structure.

In [4], an invariant is defined for 4-manifolds with contact boundary, using the Seiberg-Witten equations. According to Theorem 1.1 of [4], the existence of the symplectic structure $\omega$ on $X$ ensures that the invariant is non-trivial for this particular 4-manifold. What this means is that a variant of the 4-dimensional Seiberg-Witten equations has a non-empty space of solutions on a complete Riemannian 4-manifold $X^{+}$obtained from $X$ by adding open cones to the two boundary components. The Spin ${ }^{c}$ structure on $X^{+}$which supports these solutions has first Chern class equal to $e(\mathcal{F})$. Furthermore, the non-empty solution space persists under any compactly-supported deformation of the Riemannian metric.

In particular, given any metric $h$ on $Y$, we can equip $X^{+}$with a metric which contains an isometric copy of a cylinder, $[-R, R] \times(Y, h)$. Letting $R$ increase, we deduce in the limit the existence of a solution to the 3-dimensional equations on $(Y, h)$, supported by a $\operatorname{Spin}^{c}$ structure with first Chern class $e(\mathcal{F})$, as required. The details of this argument are contained in Proposition 5.6 and Lemma 5.7 of [4], in the absence of the perturbing term $\mu$ which appears there. Note that the second hypothesis of Proposition 5.6 is not relevant to part (a) of its conclusions, which is all that we are using.

Proof.[Proof of the theorems] Lemmas 3 and 4 show that the convex hull of the monopole classes is contained in the unit ball of the (possibly infinite) norm

$$
4 \pi \sup _{h}\|\alpha\|_{h} /\left\|s_{h}\right\|_{h}
$$

which in turn is contained in the unit ball of the dual Thurston norm. Proposition 5 says that the last of these polytopes is contained in the first, so all three coincide. This proves the two theorems.

\section{Further comments}

Floer homology. Our definition of 'monopole class' made the exposition rather straightforward, but is probably not generally useful. A more intelligent definition would be based on the non-vanishing of a corresponding Floer homology theory - the homology defined by the Morse theory in which the critical points are the solutions of the monopole equations on $Y$. In the case that $c_{1}(\mathfrak{c})$ is nonzero (which is really the only case that interests us here), the construction of this Floer homology is presumably straightforward. It is clear that the argument of Proposition 5 should prove a stronger statement, namely that this Floer homology is non-trivial for $\mathrm{Spin}^{c}$ structures arising from taut foliations. The material in [4] takes a small step in this direction, without defining the Floer homology.

Note that the integer obtained by counting solutions algebraically (the euler characteristic of the Floer homology) is not a strong enough invariant, and the convex hull of the classes for which this invariant is non-zero is in general strictly 
smaller. For a 3-manifold obtained by zero-surgery on a knot, the difference is the difference between the genus of the knot and the degree of its Alexander polynomial.

Reducible 3-manifolds. If $Y$ is reducible, it can be decomposed as a connected sum of manifolds $Y_{i}$, each of which is either irreducible or $S^{1} \times S^{2}$. If each of the pieces $Y_{i}$ admits a smooth, taut foliation then Proposition 5 still holds. To see this, note first that the unit ball of the dual Thurston norm is the product of the unit balls of the summands. Although one cannot take a connected sum of foliations, one can make a connected sum of contact structures [9]. Indeed, one can form a boundary connect sum $X$ of the cylinders $X_{i}=[0,1] \times Y_{i}$ at their boundary components $\{1\} \times Y_{i}$, and so form a symplectic 4-manifold having a contact 3-manifold $Y$ as one boundary component. The argument of Proposition 5 can now be used.

Thus our two theorems hold in all cases, except where one of the summands has an entirely degenerate Thurston norm and may not have a smooth, taut foliation. In fact, a foliation is not needed, except to provide a contact structure compatible with a symplectic form on some bounding 4-manifold: some summands which do not have taut foliations (spherical space forms, for example) can be dealt with by exhibiting the contact structure directly.

A typical 3-manifold for which we do not have a proof of Proposition 5 is a connected sum in which one summand is obtained by zero surgery on a knot of genus one. The taut foliation on the summand may not be smooth. In such cases, it seems likely that the theorems can be recovered by considering the Floer homology formulation, and analyzing the Floer homology of a connected sum.

Other norms. For a given Riemannian metric $h$, the $L^{2}$ norm of the harmonic representative for $\alpha$ can also be described as the infimum of the $L^{2}$ norms of all de Rham representatives. Let us define a $C^{0}$ norm on $H^{2}(Y ; \mathbb{R})$ by taking the infimum of the $C^{0}$ norms of the de Rham representatives. This still depends on a choice of $h$. Lemmas 3 and 4 can both be proved using this $C^{0}$ norm on $H^{2}$ and replacing the $L^{2}$ norm of the scalar curvature by the supremum of $-s_{h}$. For the first lemma, one uses the maximum principle to obtain

$$
|\Phi|^{2} \leq 2 \sup (-s)
$$

and hence

$$
\left|F_{\hat{A}}\right| \leq \sup (-s / 2),
$$

by a standard argument. For the second lemma, one must change the geometry a little to obtain the inequality. Rather than using a cylindrical metric, one should equip $\Sigma \times[0, r]$ with a metric which slowly shrinks to a narrow neck in the middle, so that the scalar curvature is at its maximum at the narrowest point, where the geometry approximates the cylinder. 
Area of embedded surfaces. A simple consequence of Theorem 2 in its $C^{0}$ norm version is a lower bound for the area of embedded surfaces. Let $Y$ be irreducible and suppose for simplicity that $Y$ contains no non-separating tori. The Thurston norm $|\sigma|$ of a class $\sigma \in H_{2}(Y ; \mathbb{Z})$ is then defined as the minimum of $-\chi(S)$ over all surfaces $S$ (not necessarily connected) which represent $\sigma$ and which contain no spheres. There exists a class $\alpha \in H^{2}(Y)$ with dual Thurston norm 1 satisfying

$$
\langle\alpha, \sigma\rangle=|\sigma|
$$

and according to the theorem, for any metric $h$, we can represent $\alpha$ by a form $a$ with

$$
\sup \left(-s_{h}\right) / 4 \pi \geq\|a\|_{C^{0}, h} .
$$

Integrating this inequality over the surface, one obtains

$$
\operatorname{Area}(\Sigma) \geq 4 \pi|\sigma| /\left\|s_{h}\right\|_{C^{0}}
$$

In other words, the area of any embedded surface is bounded below, in terms of the Thurston norm of the homology class it represents and the scalar curvature. The lower bound is achieved if $\Sigma$ realizes the Thurston norm and the geometry of the 3-manifold is cylindrical, with constant scalar curvature. The same result can be obtained using the existence of a stable, immersed minimal surface and the second variation inequality, see [5].

\section{References}

1. Y. M. Eliashberg and W. P. Thurston, Contact structures and foliations on 3-manifolds, Turkish J. Math. 20 (1996), 19-35.

2. D. Gabai, Foliations and the topology of 3-manifolds, J. Diff. Geom 18 (1983), 445-503.

3. P. B. Kronheimer, Minimal genus in $S^{1} \times M^{3}$, preprint.

4. P. B. Kronheimer and T. S. Mrowka, Monopoles and contact structures, Invent. Math. (1997), to appear.

5. R. Schoen and S. T. Yau, Existence of incompressible minimal surfaces and the topology of three-dimensional manifolds with nonnegative scalar curvature, Ann. of Math. 110 (1979), 127-142.

6. C. H. Taubes, More constraints on symplectic forms from Seiberg-Witten invariants, Math. Res. Lett. 2 (1995), 9-13.

7. $\quad \mathrm{SW} \Rightarrow \mathrm{Gr}$ : from the Seiberg-Witten equations to pseudo-holomorphic curves, J. Amer. Math. Soc. 9 (1996), 845-918.

8. W. P. Thurston, A norm for the homology of 3-manifolds, Mem. Amer. Math. Soc. 59 (1986), i-vi and 99-130.

9. A. Weinstein, Contact surgery and symplectic handlebodies, Hokkaido Math. J. 20 (1991), 241-251.

10. E. Witten, Monopoles and four-manifolds, Math. Res. Lett. 1 (1994), 769-796.

Dept. of Mathematics, Harvard University, Cambridge, MA 02138

E-mail address: kronheim@math.harvard.edu 02139

Dept. of Mathematics, Massachusetts Institute of Technology, Cambridge, MA

E-mail address: mrowka@math.mit.edu 